\title{
OCURRENCIA DE ACCIDENTES DE TRABAJO CAUSADOS POR MATERIAL CORTO-PUNZANTE ENTRE TRABAJADORES DE EN- FERMERÍA EN HOSPITALES DE LA REGIÓN NORDESTE DE SÃO PAULO, BRASIL
}

\author{
OCCUPATIONAL ACCIDENTS CAUSED BY CUTTING AND PIERCING \\ OBJECTS AMONG NURSING WORKERS IN HOSPITALS IN THE \\ NORTHEASTERN REGION OF SÃO PAULO, BRAZIL
}

\author{
MARIA HELENA PALUCCI MARZIALE*
}

\begin{abstract}
RESUMEN
Este estudio tuvo como objetivos identificar la ocurrencia de accidentes de trabajo causados por material cortopunzante entre trabajadores de enfermería y la conducta adoptada por los hospitales frente al accidente. Investigación descriptiva realizada a través del levantamiento de los accidentes de trabajo notificados durante el año de 1999 en cuatro hospitales de la región del Nordeste del Estado de São Paulo-Brasil. Los datos fueron recolectados a través del análisis documental. Fueron notificados en el periodo estudiado 117 accidentes, de los cuales 53 fueron ocasionados por material corto-punzante. Los ayudantes de enfermería fueron los que más se accidentaron $(8,9 \%)$, seguidos por los técnicos $(2,8 \%)$, auxiliares $(2,7 \%)$ y enfermeros $(2,5 \%)$. La mayoría de los accidentes ocurren con profesionales con experiencia y con tiempo de servicio mayor de un año, en mujeres, casadas o en unión libre, con edad inferior a 40 años. La mayoría de las ocurrencias de los accidentes fue en el turno de la mañana, causado por agujas o bisturíes $(84,9 \%)$, provocando lesiones en los dedos $(77,3 \%)$. Las conductas tomadas por los hospitales demostraron falta de orientación y desconocimiento por la conducta adecuada a ser seguida.
\end{abstract}

Palabras claves: Accidentes de trabajo, lesiones corto-punzantes, trabajo, enfermería.

\begin{abstract}
This study aimed at identifying the occurrence of occupational accidents caused by cutting and piercing materials among nursing workers and the conduct adopted by hospitals with respect to accidents. It is a descriptive research carried out by evaluating occupational accidents legally notified in four hospitals in the Northeastern region of São Paulo State in 1999. Data were collected through documental observation. During the studied period, 117 accidents were reported, of which 53 had been caused by cutting and piercing materials. Nursing aids comprised the most frequently affected staff $\left(8.9 \%{ }^{\circ}\right)$, followed by technicians $(2.8 \%)$, auxiliaries $(2.7 \%)$ and nurses $(2.5 \%)$. Most of the accidents took place among professionals that had been working for longer than a year and who were females, married or living in concubinage, younger than 40 years old, during the morning shift and were caused by needles and scalpels $(84.9 \%)$, producing lesions in the fingers $(77.3 \%)$. The conducts adopted by the hospitals showed lack of guidance and knowledge concerning the adequate procedure to be followed.
\end{abstract}

Keywords: Occupational accidents, needlestick lesions, work, nursing.

Recepcionado: 28.09.2001. Aceptado: 19.03.2003.

\section{INTRODUCCIÓN}

A pesar de ser imprescindibles las condiciones de seguridad para la realización de cual- quier actividad laboral, es común encontrarnos con situaciones peligrosas, en las cuales las exigencias legales para el control de los riesgos ocupacionales no son tenidas en cuen-

*Profesor Asociado de la Escuela de Enfermería de Riberão Preto - USP, Riberão Preto, Brasil. E-mail: marziale@eerp.usp.br 
ta por negligencia, causando así accidentes y enfermedades en los trabajadores.

Dentro de las varias áreas de actuación laboral está el hospital, en donde son realizadas actividades insalubres, es decir, aquellas que por su naturaleza, condiciones o métodos de trabajo exponen al trabajador a agentes nocivos para su salud por encima de los límites de tolerancia fijados en razón de la naturaleza y de la intensidad del agente y del tiempo de exposición a sus efectos (1).

Durante la ejecución de actividades insalubres o cuando los preceptos de orden y seguridad disciplinaria no son cumplidos, pueden ocurrir enfermedades y accidentes de trabajo. Accidente de trabajo es aquel que ocurre por el ejercicio del trabajo, en el servicio de la empresa, provocando lesión corporal, perturbación funcional o enfermedad que cause la muerte, pérdida o reducción permanente o temporaria de la capacidad para el trabajo (1). Enfermedad profesional es considerada en Brasil como aquélla producida o desencadenada por el ejercicio de un trabajo peculiar a determinada actividad, y la enfermedad del trabajo es entendida como aquélla adquirida en función de condiciones especiales en que el trabajo es realizado y se relaciona directamente con él (2).

Dentro de las enfermedades del trabajo están aquellas adquiridas por medio de la sangre y los fluidos corporales, como por ejemplo la hepatitis y el síndrome de inmunodeficiencia adquirida (SIDA), las cuales pueden ser transmitidas cuando ocurren accidentes con material corto-punzante.

Se estima (3) que dentro de los profesionales de la salud afectados por accidentes de trabajo ocasionados por picadas de agujas, entre $6 \%$ y $30 \%$ de éstos pueden presentar seropositividad para el virus de la hepatitis B. El riesgo de seropositividad para el HBV (virus que causa la hepatitis B humana) después de una exposición percutánea gira entorno de $30 \%$, ya para el HIV (virus del SIDA) estos índices caen para números inferiores a $1 \%$ (4). Datos más recientes publicados por el International Health Care Worker Safety Center (5) indican tasas de seropositividad, después de la exposición ocupacional al virus $\mathrm{HIV}$, de $0,30 \%$, al $\mathrm{HBV}$ de $6 \%$ a $30 \%$ y al $\mathrm{HCV}$ de 0,5 a $2 \%$.

Aunque bajo, el riesgo de seropositividad para el HIV es real y ha asombrado a los profesionales de la salud en el ejercicio de sus actividades. En relación con la contaminación por el virus de la hepatitis, la situación es alarmante, ya que las estadísticas demuestran que el profesional de enfermería está sujeto a un riesgo 15 veces mayor de contraer la enfermedad en comparación con la población en general (6).

El panorama mundial de los casos de infección por el HIV entre profesionales del área de la salud hasta 1997 era de 264 casos. Dentro de los casos documentados, 55,4\% ocurrió en los Estados Unidos, 11,7\% en Francia, 4,2\% en Inglaterra, 4,4\% en Austria, 5,3\% en Italia, 5,3\% en España, 3,2\% en Africa del Sur y el resto distribuido por otros países (5).

En el Brasil el primer caso de SIDA ocupacional reconocido oficialmente ocurrió en 1994, después de que una auxiliar de enfermería se accidentó durante la ejecución de una punción venosa en un paciente con SIDA (7). Investigaciones aisladas han sido desarrolladas, aun así la escasez de datos generales sistematizados imposibilita conocer la magnitud real del problema en nuestro país. Esta situación se vuelve preocupante cuando se relaciona con el gran número de pacientes infectados por el virus de la hepatitis y el SIDA atendidos en los hospitales brasileños.

La posibilidad de adquisición del HIV después de una exposición ocupacional puede ser inferida por la combinación de algunos factores relacionados con el accidente, es decir: a) por la vía, profundidad, tamaño y condiciones de la inoculación, tiempo de contacto entre la fuente y el profesional; b) en relación con la fuente de infección, como viremia, uso de anti-retrovirales y etapa de la enfermedad; c) en relación con las características del profesional accidentado, como anticuerpos HLA, presencia de enfermedad de base y aun el tiempo transcurrido antes de la búsqueda de atención inicial después 
del accidente (8). La exposición es clasificada de alto riesgo cuando envuelve grandes volúmenes de sangre y altos títulos de HIV en la sangre a la que se expone; medio riesgo cuando hay grandes volúmenes de sangre de exposición, y bajo riesgo en situaciones en donde existe gran volumen de sangre y no hay altos títulos de HIV en la sangre.

El riesgo de infección por el virus $\mathrm{HBV}$ y $\mathrm{HCV}$ (causantes de hepatitis B y C, respectivamente) es diferente de acuerdo con la categoría profesional, siendo mayor en el personal de enfermería, técnicos de laboratorio y médicos, debido al hecho de que esas categorías están más expuestas a la manipulación de sangre y hemoderivados (9).

La fuente de infección está constituida por pacientes contaminados, siendo el período de transmisibilidad representado por las últimas semanas del período de incubación y un tiempo variable después de la aparición de la ictericia y la elevación de las transaminasas. Entre 5 y 10\% de los individuos contaminados se transformaron en portadores (asintomáticos), no desarrollando la enfermedad pero siendo capaces de transmitir el virus (10).

El profesional que se accidenta y los servicios de salud ocupacional deben cumplir con algunas medidas importantes inmediatamente después de la ocurrencia del accidente con material corto-punzante. Inicialmente, evaluar el accidentado lavando el área afectada con agua y jabón, si la lesión es por perforación con aguja o corte; lavar con agua cuando ocurre estornudo en nariz, boca o piel; lavar con agua o suero fisiológico si hay salpicada en ojos; si el accidente produce hemorragia, buscar atención médica inmediata; cambiar cualquier ropa mojada con fluidos corporales del paciente fuente $\mathrm{y}$, finalmente, procurar el servicio de salud ocupacional de la institución (11).

La profilaxis posterior a la exposición relacionada con el HBV es la misma indicada por el Centers for Disease Control-CDC (12) para exposiciones percutáneas o en mucosas. Vacunación y / o con la inmonoglobulina puede ser utilizada en el caso de la hepatitis B; aun así, en relación con la hepatitis $C$, no existen hasta el momento recomendaciones profilácticas.

La profilaxis pos-exposición percutánea es recomendada en las situaciones en que existe la presencia de sangre en exposiciones de alto, medio y bajo riesgo, en la exposición a otros fluidos corporales con sangre visible, otros fluidos o tejidos potencialmente infectados (13).

Después de la evaluación especializada puede ser indicado al trabajador accidentado el uso de quimioprofilaxis con antiretrovirales. Esta indicación debe ser basada en el riesgo de transmisión del HIV considerando las circunstancias y el tipo de accidente, y la decisión de usarlos o no debe ser definida entre el trabajador accidentado y el médico responsable por la evaluación (13). Cuando la quimioprofilaxis fuese indicada, deberá ser iniciada, preferencialmente, en las primeras dos horas después del accidente, tiene duración de cuatro semanas (cuando el paciente fuente es positivo o desconocido), o hasta que sea conocido el resultado de serología del paciente-fuente (14).

Ante la peligrosidad del accidente de trabajo con material corto-punzante para el equipo de enfermería nos motivamos para realizar este estudio.

\section{OBJETIVOS}

- Identificar la ocurrencia de los accidentes de trabajo ocasionados por material cortopunzante entre trabajadores de enfermería de hospitales de la región nordeste del Estado de São Paulo, Brasil;

- Identificar la conducta adoptada por los hospitales frente a la ocurrencia de los accidentes de trabajo con material corto-punzante.

\section{MATERIALES Y MÉTODOS}

Se trata de un estudio descriptivo con análisis cuantitativo de los datos realizado en cua- 
tro hospitales de la región nordeste del Estado de São Paulo, Brasil, región cuya economía está centrada en la agricultura (principalmente en el cultivo de la caña de azúcar, naranja y soya), la industria y el comercio; es un importante centro educacional de la enseñanza superior (universidades) y de salud. Población estimada de 1 millón de habitantes.

Hospital A: Institución pública universitaria de la ciudad de Ribeirão Preto, con cuadro funcional de 3.947 trabajadores, siendo 1.327 pertenecientes al equipo de enfermería, distribuidos entre las siguientes categorías: 202 enfermeros, 133 técnicos de enfermería, 918 auxiliares de enfermería y 74 ayudantes de enfermería.

Hospital B: Institución privada de la ciudad de Ribeirão Preto, con cuadro funcional de 368 trabajadores, siendo 161 pertenecientes al equipo de enfermería, distribuidos entre las siguientes categorías: 16 enfermeros, 31 técnicos de enfermería, 112 auxiliares de enfermería y 2 ayudantes de enfermería.

Hospital C: Institución filantrópica de la ciudad de Batatais, con cuadro funcional de 128 trabajadores, siendo 67 pertenecientes al equipo de enfermería, distribuidos entre las siguientes categorías: 8 enfermeros, 1 técnico de enfermería, 37 auxiliares de enfermería, 21 ayudantes de enfermería.

Hospital D: Institución filantrópica de la ciudad de Sertãozinho, con cuadro funcional de 204 trabajadores, siendo 107 pertenecientes al equipo de enfermería, distribuidos entre las siguientes categorías: 10 enfermeros, 13 técnicos de enfermería, 58 auxiliares de enfermería y 26 ayudantes de enfermería.

\section{Población}

La población estuvo compuesta por los trabajadores componentes del equipo de enfer- mería de los cuatro hospitales que durante el año de 1999 tuvieron accidentes de trabajo legalmente notificados en la Comunicación del Accidente del Trabajo - CAT $(n=117)$.

\section{Procedimientos}

Los datos fueron recolectados a través de la revisión documental en los registros de los accidentes de trabajo, través de la CAT, emitidos por los hospitales.

El proyecto de investigación fue aprobado por el Comité de Ética en Investigación del Hospital Clínicas de la Facultad de Medicina de Ribeirão Preto/USP y tuvo la autorización de los gerentes de los hospitales.

\section{RESULTADOS Y DISCUSIÓN}

En el período analizado, en los cuatro hospitales fueron notificados 117 accidentes de trabajo entre profesionales de enfermería, siendo $106(90,6 \%)$ accidentes típicos y $11(9,4 \%)$ accidentes de trayecto.

Los accidentes típicos, es decir, aquellos sufridos en el local y horario de trabajo, representan la mayoría de las ocurrencias en los cuatro hospitales $(90,6 \%)$. Los accidentes de trayecto $(9,4 \%)$ fueron ocasionados por accidentes automovilísticos acontecidos en el recorrido hacia el trabajo (ida o vuelta).

A través de la Tabla 1 puede ser observada la distribución de los accidentes típicos entre los trabajadores de enfermería y el tipo de lesión sufrida.

Dentro de los accidentes típicos de trabajo ocurridos $53(50 \%)$ provocaron lesiones corto-punzantes, 32 (30,2\%) lesiones traumáticas, tales como contusiones y luxaciones en piel y mucosas.

Lesiones percutáneas ocasionadas por material corto-punzante también fueron apuntadas en estudios anteriormente realizados (1517) como la principal causa de accidentes identificada entre trabajadores de enfermería. 
Tabla 1. Distribución de los accidentes de trabajo (típicos) ocurridos entre trabajadores de enfermería de 4 hospitales de la región nordeste del Estado de São Paulo - Brasil, según el tipo de lesión. 1999.

\begin{tabular}{|c|c|c|c|c|c|c|}
\hline \multirow{2}{*}{ Tipo de lesión } & \multirow{2}{*}{$\begin{array}{l}\text { Hospital A } \\
\mathrm{n}^{\circ}\end{array}$} & \multirow{2}{*}{$\begin{array}{c}\text { Hospital B } \\
\mathrm{n}^{\circ}\end{array}$} & \multirow{2}{*}{$\begin{array}{c}\text { Hospital C } \\
\mathrm{n}^{\circ}\end{array}$} & \multirow{2}{*}{$\begin{array}{c}\text { Hospital D } \\
\mathrm{n}^{\circ}\end{array}$} & \multicolumn{2}{|c|}{ Total } \\
\hline & & & & & $\mathrm{n}^{\mathrm{o}}$ & $\%$ \\
\hline Corto-punzante & 27 & 07 & 10 & 09 & 53 & 50,0 \\
\hline Traumática & 17 & 10 & 02 & 03 & 32 & 30,2 \\
\hline Excoriación & 09 & - & - & - & 09 & 8,5 \\
\hline Otro & 08 & 02 & - & 02 & 12 & 11,3 \\
\hline Total & 61 & 19 & 12 & 14 & 106 & 100 \\
\hline
\end{tabular}

Fuente: Ocurrencia de accidentes de trabajo causados por material corto-punzante entre trabajadores de enfermería en hospitales de la región nordeste de São Paulo, Brasil.

$\mathrm{Al}$ analizar la ocurrencia de los accidentes típicos de trabajo fue constatado que en el hospital A, 61 trabajadores sufren accidentes, representando $4,6 \%$ del total de trabajadores; en el hospital B, 19 (11,8\%) de los trabajadores; en el hospital C, $12(11,2 \%)$ de los trabajadores, y en el hospital D $14(20,9 \%)$ de los trabajadores. Los datos indican que la mayor ocurrencia de accidentes de trabajo entre el personal de enfermería ocurrió en el hospital D y la menor ocurrencia se dio en el hospital A, respectivamente, una institución filantrópica de pequeño tamaño y una institución pública de gran tamaño, siendo este último el único hospital de la muestra que poseía servicio de atención específica para el trabajador.

Con relación a los accidentes de trabajo con material corto-punzante, la ocurrencia también fue mayor en el hospital D y menor en el hospital A, conforme puede ser constatado en la Tabla 2.

Tabla 2. Distribución de los trabajadores de enfermería accidentados con material corto-punzante, en 4 hospitales de la región nordeste del Estado de São Paulo, Brasil, según el tipo de lesión. 1999.

\begin{tabular}{|lccc|}
\hline Hospital & Trabajadores de Enfermería & Accidentes con corto-punzantes \\
\hline & $\mathrm{n}$ & $\mathrm{n}$ & $\%$ \\
Hospital A & 1327 & 27 & 2,1 \\
Hospital B & 161 & 07 & 4,3 \\
Hospital C & 107 & 10 & 9,3 \\
Hospital D & 67 & 09 & 13,4 \\
Total & 1162 & 53 & 29,1 \\
\hline
\end{tabular}

Fuente: Idem Tabla 1.

Los accidentes de trabajo con material corto-punzante, debido al riesgo de infección por patógenos transmitidos por la sangre, ha estimulado la realización de varios estudios con miras a la planeación de medidas preventivas, tales como la utilización de "precauciones patrón", sistema de aislamiento de substancias corporales, equipos e instrumentos de seguridad $(4,6,10,12$, $15,18)$. A pesar de esto, debido a la falta de concientización de los trabajadores, de las gerencias de los servicios de salud, muchas veces no es efectuada la notificación de este tipo de accidente, demostrando números de ocurrencia que no coinciden con la realidad.

Los accidentes de trabajo ocurridos ocasionaron 41 días de retiro del trabajo durante el año de 1999 en los cuatro hospitales. Los accidentes ocasionados por material cortopunzante fueron responsables por 24 días de retiro, lo que nos lleva a constatar que ese tipo de accidentes muchas veces no ocasiona el retiro del trabajador de sus actividades laborales, convirtiéndose en un factor al que 
muchos trabajadores reducen en importancia y desalienta la notificación de los mismos.

Los datos obtenidos sobre algunas carac- terísticas personales de los trabajadores de enfermería accidentados con material cortopunzante se presentan a continuación.

Tabla 3. Distribución de los trabajadores de enfermería afectados por accidentes de trabajo con material cortopunzante, en 4 hospitales de la región nordeste del Estado de São Paulo, Brasil, según el sexo, estado civil y la edad. 1999.

\begin{tabular}{|c|c|c|c|c|c|c|}
\hline \multirow[t]{2}{*}{ Características } & \multirow{2}{*}{$\underset{n^{\circ}}{\text { Hospital A }}$} & \multirow{2}{*}{$\begin{array}{l}\text { Hospital B } \\
\mathrm{n}^{\circ}\end{array}$} & \multirow{2}{*}{$\begin{array}{c}\text { Hospital C } \\
n^{\circ}\end{array}$} & \multirow{2}{*}{$\begin{array}{c}\text { Hospital D } \\
n^{\circ}\end{array}$} & \multicolumn{2}{|c|}{ Total } \\
\hline & & & & & $\mathrm{n}^{\mathrm{o}}$ & $\%$ \\
\hline \multicolumn{7}{|l|}{ Sexo: } \\
\hline Masculino & 25 & 04 & 09 & 08 & 46 & 86,8 \\
\hline Femenino & 02 & 03 & 01 & 01 & 07 & 13,2 \\
\hline Total & 27 & 07 & 10 & 09 & 53 & 100 \\
\hline \multicolumn{7}{|l|}{ Estado civil: } \\
\hline Soltero & 10 & 03 & 04 & 04 & 21 & 39,6 \\
\hline Casado & 13 & 04 & 04 & 05 & 26 & 49,1 \\
\hline Separado & 04 & - & 02 & - & 06 & 11,3 \\
\hline Total & 27 & 07 & 10 & 09 & 53 & 100 \\
\hline \multicolumn{7}{|l|}{ Edad: } \\
\hline $20 \mid-30$ & 05 & 04 & 02 & 02 & 13 & 24,6 \\
\hline $30 \mid-40$ & 12 & 03 & 02 & 03 & 20 & 37,7 \\
\hline 40 I- 50 & 09 & - & 04 & 01 & 13 & 24,5 \\
\hline $50 \mathrm{I}-60$ & 02 & - & 02 & 02 & 06 & 11,3 \\
\hline 60 ।-। 70 & - & - & - & 01 & 01 & 1,9 \\
\hline Total & 27 & 07 & 10 & 09 & 53 & 100 \\
\hline
\end{tabular}

Fuente: Idem. Tabla 1.

La mayoría de los accidentados era de sexo femenino $(86,8 \%)$, mitad de los trabajadores $(50 \%)$ era casado o en unión libre y apenas 0,3\% de los trabajadores poseía edad inferior a los 40 años. Tales hallazgos son característicos de la fuerza de trabajo de enfermería en el Brasil, eminentemente femenina, casada y con edad inferior a 40 años. Estu- dios, aunque de menor amplitud de la muestra, indican que el perfil de la fuerza de trabajo de enfermería no sufrió grandes alteraciones en las ultimas décadas (1, 15-19).

A través de la Tabla 4 son presentados los datos relacionados con la categoría profesional de los trabajadores accidentados.

Tabla 4. Distribución de los trabajadores de enfermería afectados por accidentes de trabajo con material cortopunzante, en 4 hospitales de la región nordeste del Estado de São Paulo, Brasil, según la categoría del trabajador. 1999.

\begin{tabular}{|lcccccr|}
\hline \multicolumn{1}{|c}{ Trabajadores } & $\begin{array}{c}\text { Hospital A } \\
\mathrm{n}^{\text {o }}\end{array}$ & $\begin{array}{c}\text { Hospital B } \\
\mathrm{n}^{\circ}\end{array}$ & $\begin{array}{c}\text { Hospital C } \\
\mathrm{n}^{\circ}\end{array}$ & $\begin{array}{c}\text { Hospital D } \\
\mathrm{n}^{\circ}\end{array}$ & \multicolumn{2}{c|}{ Total } \\
\hline Enfermero & 04 & - & 01 & 01 & 06 & 11,4 \\
Técnico de Enfermería & 02 & 02 & - & 01 & 05 & 9,4 \\
Auxiliar de Enfermería & 19 & 05 & 03 & 04 & 31 & 58,5 \\
Ayudante de Enfermería & 02 & - & 06 & 03 & 11 & 20,7 \\
Total & 27 & 07 & 10 & 09 & 53 & 100 \\
\hline
\end{tabular}

Fuente: Idem Tabla 1. 
Los datos de la Tabla 4 aparentemente indican que los auxiliares de enfermería fueron los trabajadores que menos sufrieron accidentes con material corto-punzante; aun así, cuando se calcula el porcentaje por número total de trabajadores pertenecientes a cada categoría, se evidencia que $8,9 \%$ de los ayudantes de enfermería sufrió accidentes, bien como 2,8\% de los técnicos, 2,7\% de los auxiliares y $2,5 \%$ de los enfermeros. Los dados son presentados en la Tabla 5.

Tabla 5. Distribución de los trabajadores de enfermería y de los trabajadores de enfermería afectados por accidentes de trabajo con material corto-punzante, en 4 hospitales de la región nordeste del Estado de São Paulo, Brasil, según la categoría del trabajador. 1999.

\begin{tabular}{|lrlr|}
\hline Categoría profissional & $\begin{array}{r}\text { Trabalhadores } \\
\mathrm{n}^{\circ}\end{array}$ & \multicolumn{2}{c|}{$\begin{array}{c}\text { Trabajadores afectados } \\
\mathrm{n}^{\circ}\end{array}$} \\
\hline Ayudantes de enfermería & 123 & 11 & 8,9 \\
Técnicos de enfermería & 178 & 05 & 2,8 \\
Auxiliar de enfermería & 1.125 & 31 & 2,7 \\
Enfermeros & 236 & 06 & 2,5 \\
Total & 1.662 & 53 & 3,2 \\
\hline
\end{tabular}

Fuente: Idem Tabla 1.

Los auxiliares de enfermería representaron el mayor contingente entre las categorías estudiadas debido a las actividades laborales que ejecutan; normalmente se encuentran más expuestos al riesgo de accidentes cortopunzantes. Aun así, los ayudantes de enfermería fueron los que más se accidentaron, lo que nos lleva a reflexionar sobre la relación entre la formación profesional y la exposición al riesgo debido al tipo de actividad ejecutada y la ocurrencia de los accidentes de trabajo, es decir, cuanto menor la formación y mayor exposición, consecuentemente será mayor la probabilidad de ocurrir el accidente.

En la Tabla 6 son presentados los datos relacionados con el tiempo de servicio de los trabajadores de enfermería accidentados.

Tabla 6. Distribución de los trabajadores de enfermería afectados por accidentes de trabajo con material cortopunzante, en 4 hospitales de la región nordeste del Estado de São Paulo, Brasil, según el tiempo de servicio en el hospital. 1999.

\begin{tabular}{|ccccccc|}
\hline $\begin{array}{c}\text { Tiempo de servicio } \\
\text { (Años) }\end{array}$ & $\begin{array}{c}\text { Hospital A } \\
\mathrm{n}^{\circ}\end{array}$ & $\begin{array}{c}\text { Hospital B } \\
\mathrm{n}^{\circ}\end{array}$ & $\begin{array}{c}\text { Hospital C } \\
\mathrm{n}^{\circ}\end{array}$ & $\begin{array}{c}\text { Hospital D } \\
\mathrm{n}^{\circ}\end{array}$ & \multicolumn{2}{c|}{ Total } \\
\hline Menos de 1 & 02 & 04 & 01 & 02 & 09 & 17,0 \\
1 I-6 & 15 & 03 & 03 & 02 & 23 & 43,3 \\
6 I-11 & - & - & 01 & 02 & 03 & 5,7 \\
11 I-15 & 02 & - & - & - & 02 & 3,8 \\
15 | - 19 & 01 & - & 01 & 01 & 03 & 5,7 \\
19 | - 23 & 07 & - & 01 & 01 & 10 & 18,8 \\
Más de 23 & - & - & 02 & 01 & 03 & 5,7 \\
Total & 27 & 07 & 09 & 09 & 53 & 100 \\
\hline
\end{tabular}

Fuente: Idem. Tabla 1. 
Los datos de la Tabla 6 muestran que 17\% de los trabajadores accidentados tenía menos de 1 año de servicio en el hospital, lo que indica que en este caso el motivo del accidente puede estar asociado con la falta de experiencia y adaptación a las rutinas de trabajo; 43,3\% de los accidentados poseía entre 1 y 6 años de experiencia en el hospital y el restante $(39,7 \%)$ de los trabajadores tenía más de 6 años de tiempo de servicio en la institución y el motivo del accidente puede estar asociado en otros factores (carga del trabajo, edad, fatiga, stress).

La falta de experiencia en el trabajo fue responsable por $57 \%$ de los accidentes con material corto-punzante ocurridos con trabajadores de una institución hospitalaria (20).

En relación con el mes de ocurrencia de los accidentes con corto-punzantes, se observó que éstos se presentan durante todo el año, siendo la mayor ocurrencia en los meses de enero $(15,2 \%)$ y febrero $(13,2 \%)$ y la menor ocurrencia se dio en el mes de agosto $(1,9 \%)$.

La mayor frecuencia de accidentes cortopunzantes se registró en el turno de la mañana $(47,2 \%)$, seguidos por los turnos de la tarde $(28,3 \%)$ y de la noche $(24,5 \%)$. En relación con el período de ocurrencia de los accidentes en los turnos, se constató que 50,9\% de los accidentes corto-punzantes fue en el inicio del turno; $35,8 \%$ en el final y $13,3 \%$ en el medio de la jornada de trabajo. La mayor frecuencia de los accidentes durante el turno de la mañana y de la tarde $(22,23 \%)$ también fue constatada en otros estudios (21-23). Dentro de los motivos que pueden estar asociados con la ocurrencia de los accidentes de trabajo durante el período diurno y principalmente en el turno de la mañana, podemos destacar el mayor contingente de profesionales de enfermería de la mañana, debido a las rutinas hospitalarias relacionadas con la higiene y el confort de los pacientes y realización de exámenes, diagnósticos, mayor volumen de medicamentos y cirugías.

Dentro de los factores que pueden estar asociados con los resultados obtenidos están: doble o triple jornada de trabajo de muchos profesionales de enfermería que inician su labor ya fatigados, inadecuadas formas de organización de trabajo y exceso de actividades a ser ejecutadas.

La aguja fue el objeto responsable por el mayor número de lesiones corto-punzantes en todas las instituciones hospitalarias estudiadas, representando 58,5\% de los objetos causantes de los accidentes. El pericraneal fue el segundo mayor responsable por las lesiones; como el referido objeto puede ser también considerado como aguja, observamos que juntos representaron el 84,9\% de los objetos causales de los accidentes notificados. Las hojas de bisturíes y las cuchillas fueron objetos causantes del 11,3\% de los accidentes y las ampollas de vidrio por 3,8\% de éstos.

Los dedos fueron las partes del cuerpo más afectadas en los accidentes $(77,3 \%)$, seguida por las manos $(17 \%)$, antebrazos $(3,8 \%)$ y piernas $(1,9 \%)$, resultados esperados dado el tipo causante del accidente.

En relación con las providencias tomadas por los hospitales, se observó variación en las conductas tomadas.

El Hospital A, por poseer Servicio Especializado de Ingeniería en Seguridad y Medicina del Trabajo (SEISMT), tuvo como conducta la remisión de todos los trabajadores accidentados para atención médica en el referido servicio, en donde fue hecho el registro legalmente establecido, a través de la CAT, y en casos de accidentes con contacto con sangre y secreciones el trabajador fue remitido para valoración médica en la unidad especializada en enfermedades transmisibles e infecciosas del propio hospital.

El Hospital B tuvo como conducta, en la mayoría de los accidentes $(71,4 \%)$ ocurridos, la atención del trabajador en la propia institución, la emisión del registro legal, través de la CAT, y la remisión para la unidad especializada en enfermedades transmisibles e infecciosas del Hospital Universitario. Aun así, en $28,6 \%$ de los accidentes la conducta tomada fue apenas el registro legal del accidente y la atención en el propio hospital, no siendo hecha la valoración especializada.

La conducta tomada por el Hospital C fue 
variada, es decir, en $40 \%$ de las ocurrencias ninguna atención médica fue realizada y la conducta tomada se limitó a la emisión del registro del accidente en la CAT. En $40 \%$ de las ocurrencias el trabajador fue atendido en el propio hospital y fue emitido el registro del accidente en la CAT y en 20\% de los casos hubo atención en el hospital y emisión del registro del accidente en la CAT, así como posterior remisión a la unidad especializada en enfermedades transmisibles e infecciosas del Hospital Universitario de la ciudad de Ribeirão Preto-SP.

El Hospital D tuvo como conducta para todos los accidentes ocurridos la emisión del registro en la CAT, siendo que el 33,3\% de los trabajadores accidentados fue atendido en el propio hospital y 77,8\% de los trabajadores fue remitido al Hospital Universitario de la ciudad de Ribeirão Preto-SP.

Frente a los datos obtenidos surge el interrogante sobre una posible subnotificación de los accidentes de trabajo con material cortopunzante, sobre todo debido a la pequeña importancia dada a ese tipo de problemas por las instituciones hospitalarias y por las dificultades enfrentadas para la notificación de los accidentes. Tales aspectos merecen ser investigados en futuras investigaciones.

\section{CONCLUSIONES}

Los accidentes de trabajo con material cortopunzante en la última década se tornó objeto de estudio de investigadores en todo el mundo, debido al peligro de transmisión de infecciones a través de la sangre tales como las hepatitis B, C y el SIDA.

En los cuatro hospitales de la región de Ribeirão Preto se constató que los accidentes con corto-punzantes entre el personal de enfermería correspondieron al 50\% de los accidentes típicos notificados durante el año de 1999. Dentro de los hospitales estudiados la mayor ocurrencia se dio en una institución filantrópica mixta de pequeño tamaño $(13,4 \%)$ y la menor ocurrencia se dio en una institución pública de gran tamaño $(2,1 \%)$.
Por el hecho de ocasionar estos accidentes pequeñas lesiones percutáneas y no provocar significantes índices de ausentismo en el trabajo -en este estudio fueron responsables de apenas 41 días de trabajo perdido en los cuatro hospitales estudiados durante el año de 1999- han sido, frecuentemente, subnotificados (24).

Los accidentes con material corto-punzante ocurrieron entre trabajadores de enfermería del sexo femenino $(86,8 \%)$ y masculino $(13,2 \%)$, entre trabajadores casados o en unión libre $(49,1 \%)$ y en gran parte en trabajadores con menos de 40 años $(62,3 \%)$.

La categoría profesional más accidentada fue la de los ayudantes de enfermería $(8,9 \%)$ seguidos por los técnicos $(2,8 \%)$, auxiliares $(2, \%)$ y enfermeros $(2,5 \%)$. Todos los trabajadores tenían experiencia profesional en el hospital, superior a un año $(93 \%)$.

Los accidentes ocurrieron más en el turno de la mañana $(47,2 \%)$ y en todos los meses del año, no presentando diferencias significativas a lo largo del año.

Las agujas y los pericraneales fueron los objetos responsables por el $84,9 \%$ de los accidentes, los bisturíes $(11,3 \%)$ y ampollas $(3,8 \%)$. La parte del cuerpo más afectada por las lesiones percutáneas fueron los dedos $(77,3 \%)$.

En relación con las conductas adoptadas frente a la ocurrencia del accidente de trabajo con corto-punzantes, se constató que apenas uno de los hospitales poseía servicio de Seguridad y Medicina del Trabajo (hospital A), que la conducta tomada inicialmente fue la atención del trabajador en el propio hospital y el diligenciamiento de la Comunicación de Accidente de Trabajo (CAT) y que, no todos los casos fueron remitidos a la unidad especializada para evaluación del accidente, diagnóstico y tratamiento de posible infección por los virus HBV (hepatitis B), HVC (hepatitis C) y HIV (SIDA). Se constató la falta de orientación de algunos hospitales en relación con la adecuada conducta a ser seguida, indicando la necesidad de implementación de rutinas frente a la ocurrencia de esos accidentes y en relación con la adopción de medidas preventivas para minimi- 
zar el problema, así como también una mayor atención para la notificación de los accidentes de trabajo de esa naturaleza.

\section{REFERENCIAS BIBLIOGRÁFICAS}

1. BRASIL. Ministério do Trabalho. Gabinete do Ministro. Portaria $n^{\circ} 3214,8$ de junho de (1978) Normas Regulamentadoras. In: Segurança e medicina do trabalho, São Paulo, Brasil, Atlas, (1994) 19-274.

2. GALAFASSI, M.C. (1998). Medicina do trabalho. São Paulo, Brasil, Atlas, 161 pp.

3. MURPHY, D. (1995). The development of risk management program in response to the spread of bloodborne pathogen illnesses. J. Intrav. News: 8(65):43-47.

4. KOPFER,A.M.\& McGOVER, P.N. (1993). Transmission of HIV via a needlestick injury Practive recomendations and research implications. AAOHN Journal: 41(8):374-381.

5. INTERNATIONAL HEALTH CARE WORKERSAFETY CENTER (1999). Risk of infection: following a single HIV, HBV or HCV - contaminated needlestick or sharp instrument injury. University of Virginia, Virginia, EUA (online)- http:// www.medvirginia.edu. (acessado 03/2002).

6. AMARAL, F.\& HENRIQUES, F. (1997). Picada acidental. Rev. Sinais Vitais, Lisboa, Portugal. 11:4647.

7. SÃO PAULO (29/12/1999) .Secretaria do Estado da Saúde. Programa DST/AIDS. São Paulo, Brasil, Ofício No $141 / 99$

8. SÃO PAULO (1998). Secretaria do Estado da Saúde. Programa Estadual DST/AIDS. Atualidades em DST / AIDS: Biossegurança, São Paulo, Brasil, 75 pp.

9. FERNANDES, J.V. (1999). Prevalência de marcadores sorológicos do vírus da hepatite B em trabalhadores do serviço hospitalar. Rev. Saúde Pública. São Paulo, Brasil:33(2):122-8.

10. WILLIANS, C.O. (1994). Variables influencing worker compliance with universal precautions in the emergency department. Am J. Infect Control. v. 22:138-148.

11. MULLINS, N. \& LEE, H.H.J. (1998). Occupational exposure to HIV, hepatitis B, hepatitis C and tuberculosis. Clinics pediatric medicine and surgey. 15(2):363-79.

12. CENTERS FOR DISEASE CONTROL AND PREVENTION-CDC HIV / AIDS (1996). Surveillance report. Atlanta, EUA. v. 6.

13. HENDERSON, D.K. \& GERBERDING, J.L. (1989). Prophilacticzidovudine after occupational to the human immunodeficiency virus: an interim analysis. J. Infect. Dis. 160:3210027.
14. BRASIL. Ministério da Saúde. Secretaria de Políticas de Saúde, Coordenação Nacional de DST e AIDS. Manual de condutas - Exposição ocupacional a material biológico: hepatite e HIV. Brasília, Brasil, 1999, 20 pp.

15. SILVA, A. (1995). Estudo sobre os acidentes de trabalho ocorridos com a equipe de enfermagem em unidade de centro cirúrgico de um hospital geral. In: CONGRESSO BRASILEIRO DE ENFERMAGEM EM CENTRO CIRÚRGICO, 2, São Paulo, Brasil. Anais. Sociedade Brasileira de Enfermagem de Centro Cirúrgico, pp. 94-102.

16. DIAS, C.L. (2000). Acidentes de trabalho com exposição à agentes biológicos: um estudo na FANEMA. In: CONGRESSO BRASILEIRO DE ENFERMAGEM, 52, Recife, Brasil. Livro de Resumos. Sociedade Brasileira de Enfermagem, p. 620.

17.SHIMIZU, H.E.; RIBEIRO, E.J.G.; RODRIGUES, I.P. Ocorrência de acidentes de trabalho por material pérfuro-cortante em estudantes e trabalhadores de saúde de um hospital escola do Distrito Federal. In: CONGRESSO BRASILEIRO DE ENFERMAGEM, 52, Recife, Brasil. Livro de Resumos. Sociedade Brasileira de Enfermagem, p. 621.

18. PEREIRA, T.M. (1999). Avaliação da adoção das medidas de precauções padrão em categorias específicas de profissionais de saúde. Revista eletrônica de Enfermagem. (online), - http: / / www.fen.ufg.br / revista. Goiânia, Brasil. 1(1) out./ dez.

19. LAUTERT, L. (1995). O desgaste profissional do enfermeiro. Salamanca, Espanha. 276 p. Tese (Doutorado) - Universidad Pontificia Salamanca.

20. CHAN, P. (1998). Health care worker: analysis of 113 cases. In: WORLD AIDS CONFERENCE, 12, Geneva, 1998. Abstracts. Conference Geneva, p. 408.

21.BRANDI, S. (1998). Ocorrência de acidentes do trabalho por material pérfuro-cortante entre trabalhadores de enfermagem de um hospital universitário da cidade de Campinas, Estado de São Paulo. Rev. Esc. Enfermagem, São Paulo, Brasil. 32(2)124-133.

22. VALENZUELA, S.V. (1999). Contribuição ao estudo sobre acidentes de trabalho que acometem os trabalhadores de enfermagem em hospitais chilenos. Ribeirão Preto, São Paulo, Brasil, 183p. Tese (Doutorado) - Escola de Enfermagem de Ribeirão Preto/Universidade de São Paulo.

23. GODFR, K. (2001). Sharp practice. Nursing Times, 97(3):22-24.

24. NAPOLEÃO, A.A. (1999). Causas de subnotificação de acidentes de trabalho: visão dos trabalhadores de um hospital do interior paulista. Ribeirão Preto, São Paulo, Brasil, 122 p., Dissertação (Mestrado), Escola de Enfermagem de Ribeirão Preto/ Universidade de São Paulo. 